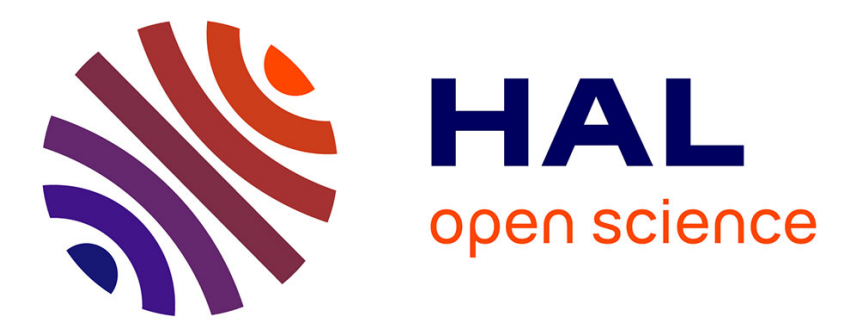

\title{
Flatness-Based Control of Electrostatically Actuated MEMS With Application to Adaptive Optics: A Simulation Study
}

Guchuan Zhu, Jean Lévine, Laurent Praly, Yves-Alain Peter

\section{To cite this version:}

Guchuan Zhu, Jean Lévine, Laurent Praly, Yves-Alain Peter. Flatness-Based Control of Electrostatically Actuated MEMS With Application to Adaptive Optics: A Simulation Study. Journal of Microelectromechanical Systems, 2006, 15 (5), pp.1165-1174. 10.1109/JMEMS.2006.880198 . hal00492754

HAL Id: hal-00492754

https://hal-mines-paristech.archives-ouvertes.fr/hal-00492754

Submitted on 16 Jun 2010

HAL is a multi-disciplinary open access archive for the deposit and dissemination of scientific research documents, whether they are published or not. The documents may come from teaching and research institutions in France or abroad, or from public or private research centers.
L'archive ouverte pluridisciplinaire HAL, est destinée au dépôt et à la diffusion de documents scientifiques de niveau recherche, publiés ou non, émanant des établissements d'enseignement et de recherche français ou étrangers, des laboratoires publics ou privés. 


\title{
Flatness-Based Control of Electrostatically Actuated MEMS With Application to Adaptive Optics: A Simulation Study
}

\author{
Guchuan Zhu, Jean Lévine, Laurent Praly, and Yves-Alain Peter, Member, IEEE
}

\begin{abstract}
Typical adaptive optics (AO) applications require continual measurement and correction of aberrated light and form closed-loop control systems. One of the key components in microelectromechanical system (MEMS) based AO systems is the parallel-plate microactuator. Being electrostatically actuated, this type of devices is inherently instable beyond the pull-in position when they are controlled by a constant voltage. Therefore extending the stable travelling range of such devices forms one of the central topics in the control of MEMS. In addition, though certain control schemes, such as charge control and capacitive feedback, can extend the travelling range to the full gap, the transient behavior of actuators is dominated by their mechanical dynamics. Thus, the performance may be poor if the natural damping of the devices is too low or too high. This paper presents an alternative for the control of parallel-plate electrostatic actuators, which is based on an essential property of nonlinear systems, namely differential flatness, and combines the techniques of trajectory planning and robust nonlinear control. It is, therefore, capable of stabilizing the system at any point in the gap while ensuring desired performances. The proposed control scheme is applied to an $\mathrm{AO}$ system and simulation results demonstrate its advantage over constant voltage control.

[1613]
\end{abstract}

Index Terms-Adaptive optics (AO), deformable mirrors (DM), electrostatic actuator, flat systems, microelectromechanical systems (MEMS), robust nonlinear control, trajectory planning.

\section{INTRODUCTION}

A PPLICATIONS like free space optical communication and targeting require smart systems, able to adapt to changing conditions (e.g., air turbulence, atmospheric perturbations, temperature, mechanical stress, etc.). Adaptive optics (AO) is a technique that can correct aberrations, varying both spatially and temporally in real time and, hence, it is particularly well suited to respond to this requirement.

The basic concept of $\mathrm{AO}$ is shown in Fig. 1 and was first proposed by the astronomer Babcock [2] in 1953. In this

Manuscript received June 8, 2005; revised December 20, 2005. This work was supported in part by École Polytechnique de Montréal under a program of start-up funds. Subject Editor H. Zappe.

G. Zhu is with the Department of Electrical Engineering, École Polytechnique de Montréal, Montréal, QC H3C 3A7, Canada (e-mail: guchuan.zhu@ polymtl. ca).

J. Lévine and L. Praly are with the Centre Automatique et Systèmes, École des Mines de Paris, 35 rue Saint Honoré, 77305 Fontainebleau cedex, France (e-mail: jean.levine@nsmp.fr; laurent.praly@nsmp.fr).

Y-A. Peter is with the Engineering Physics Department, École Polytechnique de Montréal, Montréal, QC H3C 3A7, Canada (e-mail: yves-alain. peter@polymtl.ca).

Digital Object Identifier 10.1109/JMEMS.2006.880198 system, the aberrated light from a telescope is reflected off the deformable mirror (DM). A small fraction of light is split to the wavefront sensor (WFS). The optical aberrations in the incoming wavefront are sent to the wavefront analyzer (WFA), which generates the desired configuration of reflecting surface. The control unit computes then the appropriate voltage commands which are feedback to the DM. Based on the control signals, the DM changes its shape and provides the conjugate corrections to the optical distortions. Clearly, the typical configuration of $\mathrm{AO}$ forms a closed-loop control system. Babcock's AO system was required to remove the deleterious aberrations introduced by the earth's atmosphere when using large ground-based telescopes. For astronomy, the distortion is introduced mainly by the Earth's atmosphere, but any application (e.g., vision science [1]) where there is an intervening source of aberration can benefit from AO.

Conventional adaptive mirrors are complex, heavy weighted, large in size, and expensive [3]. In a drive to reduce their size and cost, microelectromechanical deformable mirrors (MEM-DM) have recently been developed. Due to its high functionality, low mass, low power consumption, and low manufacturing cost, MEM-DM is an excellent fit for many applications, e.g., space applications, which require a low launch mass. Three types of MEM-DM using silicon surface and bulk micromachining are currently being pursued [4]: continuous face sheet mirrors backed by individual actuating elements [5], microfabricated membrane mirrors [3], [6], and segmented mirrors [7]-[9]. The choice for a specific type of MEM-DM depends on the application [10]. Continuous membrane mirrors have optimal fill factor and no diffraction effects. However, they suffer from crosstalk, have limited deformation range, and are slower than segmented mirrors. Continuous membrane mirrors are used when high power dissipation is an issue (e.g., laser micromachining) and when high-order corrections are needed (e.g., astronomy). Segmented mirrors are preferred for high speed applications (e.g., optical free space communication). All of the cited mirrors are actuated by parallel-plate electrostatic actuators. These types of actuators can be easily integrated under the mirror, without compromising the fill factor. Electrostatic comb drive actuators are also used in AO systems [9]. Microfabrication of this type of devices is much more complex and they are only used when additional large tip/tilt angles are required [9].

A common feature provided by the above mentioned MEM-DMs is that a desired configuration of reflecting surface can be produced by an underlying two-dimensional (2-D) array 


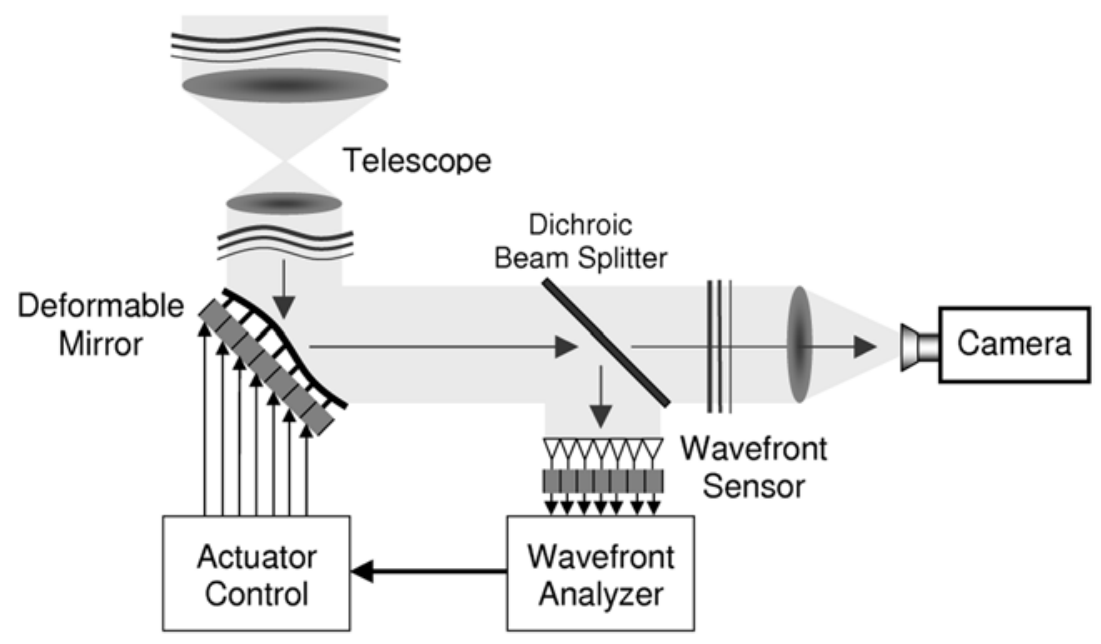

Fig. 1. Basic principle of adaptive optics for astronomical application with closed-loop actuation control (adapted from Fig. 1 in [1]).

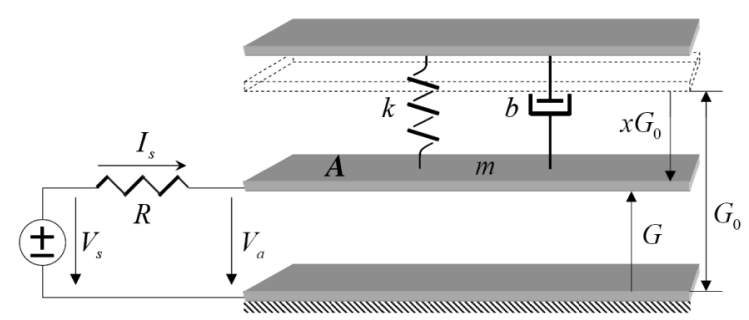

Fig. 2. Scheme of 1DOF parallel-plate electrostatic actuator.

of microdevices, each of them making piston motion. When the microdevices are actuated by electrostatic force, they can be modeled as one degree of freedom (1DOF) parallel-plate electrostatic actuator, whose scheme is shown in Fig. 2. The mechanical part of the actuator can be represented as a spring-mass-dashpot assembly, for which $m, b$, and $k$ are the mass of the moveable upper electrode, the damping coefficient, and the elastic constant, respectively. In this figure, $G(t)$ is the air gap, $G_{0}$ the zero voltage gap, $x$ the normalized deflection, and $A$ the plate area. The actuator is driven by a voltage source, where $I_{s}(t), V_{s}(t)$, and $V_{a}(t)$ are the source current, the applied voltage, and the actuation voltage, respectively. Finally, $R$ represents the resistance of the device. This paper will address the control of individual actuator, while the control of MEM-DM in the context of networked environment will be considered in a separate work.

The simplest control of electrostatically actuated microdevices might be to apply a constant voltage, whose value is deduced from the static relationship between the actuation voltage and the position of moveable plate. However, with such an open-loop static control scheme the stable traveling range of the moveable plate is limited to the one third of its full gap, the distance between the top plate and the bottom electrode when the voltage across the device equals zero [11]. Beyond that point, the moveable plate will suddenly and catastrophically snap down to the fixed electrode, rapidly reducing the gap to zero. This phenomenon is known as "pull-in." If the goal is to achieve a stable traveling range of $G_{0}$, a simple solution is to extend the air gap to $3 G_{0}$. Note that the additional $2 G_{0}$ gap can be implemented by inserting an equivalent series capacitor [12], [13]. An equilibrium beyond the pull-in position can eventually be stabilized by a closed-loop feedback control [14]. Recent researches have shown that adding series capacitor acts as capacitive feedback, which is effectively a special case of input-output linearization feedback control, when the charge is chosen as the output [15], [16].

Besides stabilizing the actuator around the set-point of operations, many MEMS-based applications impose stringent requirements on the transient behavior of actuator, such as settling time, overshoot and oscillation. Furthermore, the control schemes should also be robust vis-à-vis manufacturing tolerance, operation points, modeling errors, parameter uncertainties, and environmental disturbances. It is indeed a very complex task to incorporate all the aforementioned factors into the design of control algorithms under the framework of linear control theory, and compromising the optimality of the system is inevitable [17]. This motivates the application of nonlinear control techniques to improve the overall performance for MEMS devices.

This paper will present a method for the design of control systems for electrostatically actuated parallel-plate microdevices, which can meet different performance requirements regardless of specific configurations. The considered problem is set-point control from any point in the gap to any other points between the electrodes. The controller design uses the method presented in [18], which combines the techniques of trajectory planning and robust nonlinear control. More precisely, based on differential flatness [19], [20], a feasible reference trajectory is constructed first in the phase plane, ensuring fast rise time while having well damped transient response. Then a robust closed-loop feedback control obtained by the potential method [21], [22] is added to the control scheme, making the reference trajectory an attractive invariant manifold.

The rest of the paper is organized as follows. Section II presents the model of a 1DOF, parallel-plate electrostatic actuator. Section III demonstrates that such a system is differentially flat and, based on this property, an open-loop control under the framework of trajectory planning is constructed. In Section IV, a closed-loop control is proposed, while a reduced 
order observer, required for implementing the proposed control scheme due to the lack of speed measurement, is presented in Section V. Finally, the simulation results are reported in Section VI and Section VII contains some conclusions.

\section{Modeling OF Electrostatic ACtUAtor}

Consider now the MEMS device shown in Fig. 2. Letting $Q(t)$ be the charge on the device and denoting by $\varepsilon$ the permittivity in the gap, then the capacitance of the device is

$$
C(t)=\frac{\varepsilon A}{G(t)}
$$

and the attractive electrostatic force on the moving plate is

$$
F(t)=\frac{V_{a}^{2}}{2} \frac{\partial C}{\partial G}=-\frac{\varepsilon A V_{a}^{2}}{2 G^{2}(t)}=-\frac{Q^{2}(t)}{2 \varepsilon A} .
$$

Thus, the equation of motion of the actuator is given by

$$
m \ddot{G}(t)+b \dot{G}(t)+k\left(G(t)-G_{0}\right)=-\frac{Q^{2}(t)}{2 \varepsilon A} .
$$

From (2) and (3) it can be seen that the electrostatic force $F$ increases with the inverse square of gap, while restoring mechanical force [the third term in the left-hand side (LHS) of (3)] increases linearly with the plate deflection. The critical value for the voltage across the device is the pull-in voltage, given by (see, e.g., [11])

$$
V_{p i}=\sqrt{\frac{8 k G_{0}^{2}}{27 C_{0}}}
$$

for that if the equilibrium voltage $V_{a}^{*}<V_{p i}$, there are two equilibrium points in the gap, lying in $\left[0,(2 / 3) G_{0}\right]$ and $\left[(2 / 3) G_{0}, G_{0}\right)$, respectively. Whereas if $V_{a}^{*}=V_{p i}$, there is only one equilibrium point at the one third of the gap. And finally, if $V_{a}^{*}>V_{p i}$, then there is no equilibrium, and the system is dominated by the electrostatic force. The equilibrium at the one third of the gap is the so-called pull-in position and the corresponding equilibrium voltage, $V_{p i}$, the pull-in voltage. It can be shown that all equilibria beyond the pull-in position are unstable (see, e.g., [11]). Therefore, in terms of bifurcation theory, this critical point corresponds to a saddle-node bifurcation with respect to equilibrium voltage. Consequently, with a constant voltage control, the traveling range of the actuator is limited to the one third of its full gap.

Assuming the system started operating from an initially uncharged state at $t=0$, then the charge on the electrodes at the time $t$ is

$$
Q(t)=\int_{0}^{t} I_{s}(\tau) d \tau
$$

or equivalently

$$
\dot{Q}(t)=I_{s}(t)
$$

The current through the resistor $R$ can be obtained by a simple application of Kirchhoff's Voltage Law and reads [11]

$$
\dot{Q}(t)=\frac{1}{R}\left(V_{s}(t)-\frac{Q(t) G(t)}{\varepsilon A}\right) .
$$

To make the system analysis and control design easier, we transform the system (3)-(7) into normalized coordinates by changing the time scale, $\tau=\omega_{0} t$, and performing a normalization as follows [23] :

$$
\begin{aligned}
& x=1-\frac{G}{G_{0}}, \quad q=\frac{Q}{Q_{p i}} \\
& u=\frac{V_{s}}{V_{p i}}, \quad i=\frac{I_{s}}{V_{p i} \omega_{0} C_{0}}, \quad r=\omega_{0} C_{0} R
\end{aligned}
$$

where $C_{0}=\varepsilon A / G_{0}$ is the capacitance at rest, $Q_{p i}=$ $(3 / 2) C_{0} V_{p i}$ the pull-in charge corresponding to the pull-in voltage, $\omega_{0}=\sqrt{(k / m)}$ the undamped natural frequency, and $\zeta=\left(b / 2 m \omega_{0}\right)$ the damping ratio.

Accordingly, the normalized voltage across the actuator can be expressed in terms of the normalized deflection of the moveable electrode

$$
u_{a}=\frac{3}{2} q(1-x)
$$

and the dynamics of normalized charge becomes

$$
\dot{q}=\frac{2}{3} i
$$

Let $v=\dot{x}$ be the speed of deflection of the moveable electrode, then the system (3) and (7) can be written in the normalized coordinates as

$$
\left\{\begin{array}{l}
\dot{x}=v \\
\dot{v}=-2 \zeta v-x+\frac{1}{3} q^{2} \\
\dot{q}=-\frac{1}{r} q(1-x)+\frac{2}{3 r} u
\end{array}\right.
$$

which is defined on the state space $\chi=\left\{(x, v, q) \subset \mathbb{R}^{3} \mid x\right.$ $\in[0,1], q \geq 0\}$. Note that the normalized deflection is limited to 1 . In the case where the thickness of the insulating material coated on the bottom plate should be taken into account [15], [16], it suffices to incorporate this factor with the variable transformation while normalizing the deflection.

It can be seen that in the new coordinates, the mechanical subsystem has a damping ratio $\zeta$ and a undamped natural frequency of 1 .

Since the system analysis and control design will be performed in the normalized coordinates, we can use $t$ to denote the time and omit the qualifier "normalized," if no confusion will be introduced.

\section{Flatness AND TRAJECTORY PlanNING}

Like many mechatronic systems, electrostatically actuated MEMS are differentially flat. In fact, from the second equation in (11), we have

$$
q=\sqrt{3(\ddot{x}+2 \zeta \dot{x}+x)}
$$


Furthermore, the third-order time derivative of $x$ is

$$
x^{(3)}=-2 \zeta \ddot{x}-\dot{x}+\frac{2}{3} q \dot{q}
$$

and, hence, the input to the system can be expressed as

$$
u=\frac{3 r}{2}\left(\frac{3}{2 q}\left(x^{(3)}+2 \zeta \ddot{x}+\dot{x}\right)+\frac{1}{r} q(1-x)\right)
$$

Therefore, all the states, as well as the input in (11) can be obtained from $x$ and its time derivatives, $\dot{x}, \ddot{x}$, and $x^{(3)}$, and the system (11) is differentially flat with $x$ as flat output [19], [20].

We remark that if the reference trajectory is such that $y_{r}$ has a monotonic behavior then we can use $x$ itself as a parameter instead of time. Moreover, (11) can be written, by eliminating the time, as

$$
\left\{\begin{array}{l}
v v^{\prime}=-2 \zeta v-x+\frac{1}{3} q^{2} \\
v q^{\prime}=-\frac{1}{r} q(1-x)+\frac{2}{3 r} u
\end{array}\right.
$$

where $v^{\prime}=(d v / d x)$ and $q^{\prime}=(d q / d x)$. Thus, we have

$$
q^{2}=3 v v^{\prime}+6 \zeta v+3 x
$$

and

$$
u=\frac{3}{2}\left(r v q^{\prime}+q(1-x)\right)
$$

Noting that

$$
q^{\prime}=\frac{3}{2 q}\left(2 \zeta^{\prime}+v^{2}+v v^{\prime \prime}+1\right)
$$

is a function of $v, v^{\prime}$ and $v^{\prime \prime}$, with $v^{\prime \prime}=\left(d v^{\prime} / d x\right)=\left(d^{2} v / d x^{2}\right)$. This proves that $q$ and $u$ can be expressed as functions of $v, v^{\prime}$ and $v^{\prime \prime}$, and thus the system (15) is flat with respect to $v$ (now a function of $x$ ). Strictly speaking, the system (15) is orbitally flat, due to the fact that it is parameterized with respect to $x$ [20].

Since the system (15) is flat, it is possible to compute any trajectory of the system without integrating the corresponding differential equations.

It is now convenient to denote by $\theta, \rho$ and $\mu$ the functions $x \mapsto v, x \mapsto q$, and $x \mapsto u$, respectively, to avoid confusion with the time functions. The trajectory planning problem now consists in determining the curve $x \mapsto \theta(x)$.

To bring the system from the initial point $x_{i}$ to a desired point $x_{f}$ in the phase plane, one only needs to find a sufficiently smooth trajectory $x \mapsto \theta(x)$, such that the initial and final conditions are all met. Since the trajectory $x \mapsto \theta(x)$ does not need to verify any differential equations, it can be simply constructed, for example, by polynomial interpolations. For example, if $v\left(x_{i}\right), v^{\prime}\left(x_{i}\right), v^{\prime \prime}\left(x_{i}\right), v\left(x_{f}\right), v^{\prime}\left(x_{f}\right)$, and $v^{\prime \prime}\left(x_{f}\right)$ are known, the desired trajectory $x \mapsto \theta(x)$ can be expressed as a fifth degree polynomial [18]

$$
\begin{aligned}
\theta(x)=a_{0}+a_{1} \xi(x)+ & a_{2} \xi^{2}(x) \\
& +a_{3} \xi^{3}(x)+a_{4} \xi^{4}(x)+a_{5} \xi^{5}(x)
\end{aligned}
$$

where $\xi(x)=\left(\left(x-x_{i}\right) / X\right)$ with $X=x_{f}-x_{i}$. The coefficients in (19) can be obtained by applying the initial and final conditions and the results are

$$
\begin{aligned}
a_{0}= & 0, \quad a_{1}=X v^{\prime}\left(x_{i}\right), \quad a_{2}=\frac{1}{2} X^{2} v^{\prime \prime}\left(x_{i}\right) \\
a_{3}= & -6 X v^{\prime}\left(x_{i}\right)-\frac{3}{2} X^{2} v^{\prime \prime}\left(x_{i}\right) \\
& -4 X v^{\prime}\left(x_{f}\right)+\frac{1}{2} X^{2} v^{\prime \prime}\left(x_{f}\right) \\
a_{4}= & 8 X v^{\prime}\left(x_{i}\right)+\frac{3}{2} X^{2} v^{\prime \prime}\left(x_{i}\right) \\
& +7 X v^{\prime}\left(x_{f}\right)-X^{2} v^{\prime \prime}\left(x_{f}\right) \\
a_{5}= & -3 X v^{\prime}\left(x_{i}\right)-\frac{1}{2} X^{2} v^{\prime \prime}\left(x_{i}\right) \\
& -3 X v^{\prime}\left(x_{f}\right)+\frac{1}{2} X^{2} v^{\prime \prime}\left(x_{f}\right) .
\end{aligned}
$$

Since

$$
\begin{aligned}
& \dot{v}=v v^{\prime} \\
& \ddot{v}=2 v v^{\prime 2}+v^{2} v^{\prime \prime}
\end{aligned}
$$

the initial and final constraints on the trajectory will be respected as long as

$$
\begin{aligned}
\left|v^{\prime}\left(x_{i}\right)\right| & <\infty, \quad\left|v^{\prime}\left(x_{f}\right)\right|<\infty, \quad\left|v^{\prime \prime}\left(x_{i}\right)\right|<\infty \\
\left|v^{\prime \prime}\left(x_{f}\right)\right| & <\infty .
\end{aligned}
$$

The free parameters $v^{\prime}\left(x_{i}\right), v^{\prime \prime}\left(x_{i}\right), v^{\prime}\left(x_{f}\right)$, and $v^{\prime \prime}\left(x_{f}\right)$ can be chosen, for example, to make the reference trajectory to fit performance specifications, under constraints on drive current and applied voltage.

Note that since all the time derivatives of $x$ have to vanish at the equilibria, the desired trajectory can be represented by a polynomial with an arbitrary, finite order. This allows the addition of more degrees of freedom in trajectory tuning and the obtaining of the desired behavior (e.g., fast rise time, low overshoot, and well damped oscillations).

Finally, it is worth pointing out that the pull-in equilibrium point is removed in this approach since we are using time-varying controls that exactly generate the required polynomial reference trajectory of $v(x)$. This remark will even be enhanced in Section IV by a closed-loop synthesis that makes the reference trajectory tracking stable.

\section{CONTROL SYNTHESIS}

A closed-loop control is required in our scheme in order to stabilize the system along any reference trajectory. In addition, the closed-loop control will make the system robust vis-à-vis modeling errors, parameter uncertainties, and disturbances. Obviously, the function $(\theta, \rho)$ previously designed defines an invariant set of (11). Indeed, for any initial condition $(x, v, q)$ satisfying

$$
(v, q)=(\theta(x), \rho(x))
$$

the input

$$
u=\mu(x)
$$




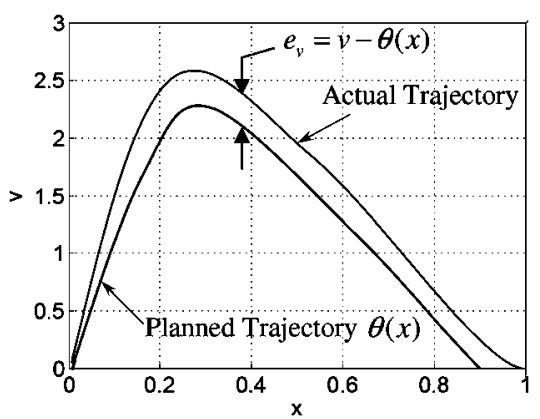

Fig. 3. Invariant manifold and its distance to out-of-the-manifold points.

satisfies (11) and is such that the corresponding solution $(x(t), v(t), q(t))$ satisfies for all $t$

$$
(v(t), q(t))=(\theta(x(t)), \rho(x(t))) .
$$

Moreover, this trajectory coincides with the planned reference trajectory.

On this set, the dynamics of (11) reduce to

$$
\dot{x}=\theta(x) \text {. }
$$

Outside this set, we have

$$
\dot{x}=v=\theta(x)+(v-\theta(x))=\theta(x)+e_{v}
$$

where $e_{v}$ is the distance between the invariant manifold and offthe-manifold points, as shown in Fig. 3.

Thus, in order to ensure that the trajectory of the dynamic system (11) converges to the planned reference trajectory, it suffices to asymptotically stabilize the invariant set $v=\theta(x)$. According to (15)

$$
\begin{aligned}
\dot{e}_{v}= & \left(-2 \zeta v-x+\frac{1}{3} q^{2}\right)-\theta^{\prime}(x) v \\
= & \theta(x) \theta^{\prime}(x)-\frac{1}{3} \rho^{2}(x)+2 \zeta \theta(x)+x \\
& -2 \zeta v-x+\frac{1}{3} q^{2}-\theta^{\prime}(x) v \\
= & -\left(2 \zeta+\theta^{\prime}(x)\right) e_{v}-\frac{1}{3}(\rho(x)+q)(\rho(x)-q) .
\end{aligned}
$$

By adding and removing a term $(1 / 3) g(x) e_{v}(\rho(x)+q)$ to and from (26), we obtain

$$
\begin{aligned}
\dot{e}_{v}=-\left(2 \zeta+\theta^{\prime}(x)\right. & \left.+\frac{1}{3} g(x)(\rho(x)+q)\right) e_{v} \\
& -\frac{1}{3}(\rho(x)+q)\left(\rho(x)-q-g(x) e_{v}\right) .
\end{aligned}
$$

In order to stabilize (27), we impose

$$
\dot{e}_{q}=-k_{0} e_{q}+\frac{1}{3}(\rho(x)+q) e_{v}
$$

where

$$
e_{q}=\rho(x)-q-g(x)(v-\theta(x)) .
$$

Consider now the following Lyapunov function candidate

$$
V(x, v, q)=\frac{1}{2} e_{v}^{2}+\frac{1}{2} e_{q}^{2} .
$$

The time derivative of $V(x, v, q)$ along the solutions of (27) and (28) is

$$
\dot{V}(x, v, q)=\dot{e}_{v} e_{v}+\dot{e}_{q} e_{q}=-\eta(x, q) e_{v}^{2}-k_{0} e_{q}^{2}
$$

where

$$
\eta(x, q)=2 \zeta+\theta^{\prime}(x)+\frac{1}{3} g(x)(\rho(x)+q) .
$$

Since $\rho(x)$ is positive for all $x \in(0,1]$ and $q$ is nonnegative, by choosing $g(x)$ as a constant given by

$$
g_{0} \geq 3 \sup _{x \in(0,1]}\left(\frac{\left|\theta^{\prime}(x)\right|}{\rho(x)}\right)
$$

$\eta(x, q)$ will be a positive function. It is clear that $\dot{V}(x, v, q)$ will be negative definite for any $k_{0}>0$. Hence the error dynamics (27) and (28) are asymptotically stable provided that $g_{0}$ is chosen as (30) and $k_{0}>0$, and the reference trajectory is an asymptotically attractive one.

Finally, the control signal can be deduced from (28), which is given as

$$
\begin{aligned}
u=\mu(x)+\frac{3 r}{2}( & \left(k_{0}+\frac{1}{3} g_{0}(\rho(x)+q)\right) e_{q} \\
& +\frac{1}{r}(1-x)(q-\rho(x)) \\
& +\left(g_{0}\left(2 \zeta+\theta^{\prime}(x)+\frac{1}{3} g_{0}(\rho(x)+q)\right)\right. \\
& \left.\left.+\rho^{\prime}(x)\right) e_{v}\right) .
\end{aligned}
$$

\section{SPEEd ObSERVER DESIGN}

Usually, the charge on the device and the gap between the electrodes can be deduced from the input current, the voltage across the device, and the capacitance. However, directly sensing the velocity during the normal operation of the device is extremely difficult, if not impossible. We need, therefore, to construct a speed observer in order to provide the estimate of $v$ required for implementing the closed-loop control described in the previous section. It can be shown that (11) with the deflection and the charge as outputs admits the observer canonical form [24]. Therefore, it is possible to find a full order observer with linear error dynamics. However, we need only to directly construct a reduced order speed observer. To this aim, we set

$$
z=v-k_{v} x
$$


where $k_{v}$ is an arbitrary positive real number. Differentiating (32), we get

$$
\dot{z}=-\left(\left(2 \zeta+k_{v}\right) k_{v}+1\right) x-\left(2 \zeta+k_{v}\right) z+\frac{1}{3} q^{2} .
$$

Thus, if we set

$$
\hat{z}=\hat{v}-k_{v} x
$$

where $\hat{v}$ is the required estimate of $v$ and $\hat{z}$ the estimate of $z$, then

$$
\dot{\hat{z}}=-\left(\left(2 \zeta+k_{v}\right) k_{v}+1\right) x-\left(2 \zeta+k_{v}\right) \hat{z}+\frac{1}{3} q^{2} .
$$

Let $e=\hat{z}-z=\hat{v}-v$ denote the estimation error, and note that $(d / d t)(\hat{z}-z)=(d / d t)(\hat{v}-v)=\dot{e}$. The error dynamics can be deduced from (33) and (34)

$$
\dot{e}=-\left(2 \zeta+k_{v}\right) e
$$

and is globally exponentially stable at the origin with a decay rate defined by $k_{v}$. This implies that

$$
\hat{v}=\hat{z}+k_{v} x
$$

and (34) form an exponential observer.

\section{SimULATION RESUltS}

To illustrate the performance of the proposed control scheme, we consider a segmented MEM-DM presented in [25]. The device is fabricated using Multi User MEMS Process (MUMPS) [26]. The electrodes are patterned in the first polysilicon layer (poly0). The second polysilicon layer (poly1) and the third polysilicon layer (poly2) form the pads on the electrode chip. The mirrors are patterned in the second polysilicon layer (poly1), and the actuators in the third polysilicon layer (poly2) of the mirror chip. The mirror chip is then mechanically bonded to the electrode chip by gold compression bonding. The mirror array is electrically connected to a ground electrode by the bonding pads. Fig. 4 shows the top view of two actuators and the cross-section view of two micromirrors of the final structure. The micromirror is sustained by four beams clamped at the corners as shown and acts as the moving electrode.

The final bonded stacks of layers provides a $5.25 \mu \mathrm{m}$ spacing between the fixed and moving electrodes. The pixel size of each mirror is $400 \mu \mathrm{m} \times 400 \mu \mathrm{m}$. The size of the underneath electrode is $200 \mu \mathrm{m} \times 200 \mu \mathrm{m}$. The calculated elastic constant was $489 \mathrm{~N} / \mathrm{m}$, while the measured elastic constant is $388 \mathrm{~N} / \mathrm{m}$, about $80 \%$ of the calculated one 1 . This discrepancy is assumed to be due to difference in the design values and the actual dimensions of the fabricated devices. It is observed that when operating under the normal pressure, the mirror exhibited an overdamped behavior. The exact viscous damping coefficient is unknown, which is one of the main sources of uncertainty in the considered system. The pull-in voltage can be calculated from (4), which is $213.7 \mathrm{~V}$.

${ }^{1}$ Measurement provided by [25]. A similar setup is being built at the École Polytechique de Montreal, but not yet available. Only numerical simulations are considered in this work and real experiments will be presented in a future paper.
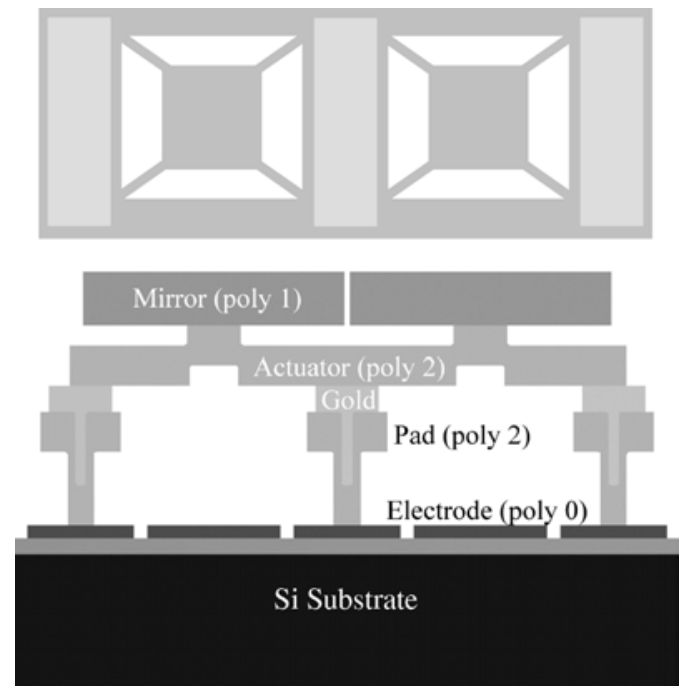

Fig. 4. Schematic top and cross-section view of 2 pixels showing the layer stack. The vertical scale is exaggerated.

Note that the algorithm for trajectory generation is time independent (see Section III) and, hence, reference trajectories can be generated in any coordinates. Obviously it is much simpler to generate the reference trajectories in the normalized coordinates and then to convert the control signals into the ones in the original coordinates. This will also make the trajectory generator generic and reusable for other MEMS devices of the same type. However, the closed-loop controller and the observer have to be implemented in the real time scale.

The nominal trajectory in the normalized coordinates is built based on the performance specifications (corresponding to a system with a damping ratio of 1.5 , driven by an ideal electrical source) and is drawn in Fig. 5(a). The function describing the displacement speed of the moveable plate with respect to the deflection is obtained by polynomial interpolation, from which $v^{\prime}\left(x_{i}\right), v^{\prime \prime}\left(x_{i}\right), v^{\prime}\left(x_{f}\right)$, and $v^{\prime \prime}\left(x_{f}\right)$ can be deduced. The reference trajectory can then be determined from (19) and (20). The reference trajectories corresponding to different deflections are shown in Fig. 5(b). It can be seen that for a full gap deflection, the maximum speed of the reference trajectory is lower than the one of the nominal trajectory. However, the simulation results show that the performance has not been significantly affected.

To help tuning the controller, the function $\theta^{\prime}(x) / \rho(x)$ [see (30)] corresponding to the planned trajectories is drown in Fig. 6. It can be seen that for $x_{\min }=10^{-3}$, or equivalently a deflection of $5.2(\mathrm{~nm})$ from the position of rest, $\sup _{x \in\left(x_{\min }, 1\right]}\left(\left|\theta^{\prime}(x)\right| / \rho(x)\right) \approx 10$. The controller gain $g_{0}$ can then be chosen to be about 30. Note that the surface roughness of the micromirror is about $40(\mathrm{~nm})$, the actuator provides then a sufficient resolution around the position of rest with this controller. Note also that the position of rest is stable for zero input, therefore, to bring the actuator back to this point it suffices to set the control signal to zero.

The control scheme has been tested with numerical simulation for a single pixel of the DM. In the configuration of the controller, the elastic constant is set to $489 \mathrm{~N} / \mathrm{m}$ (the designed 

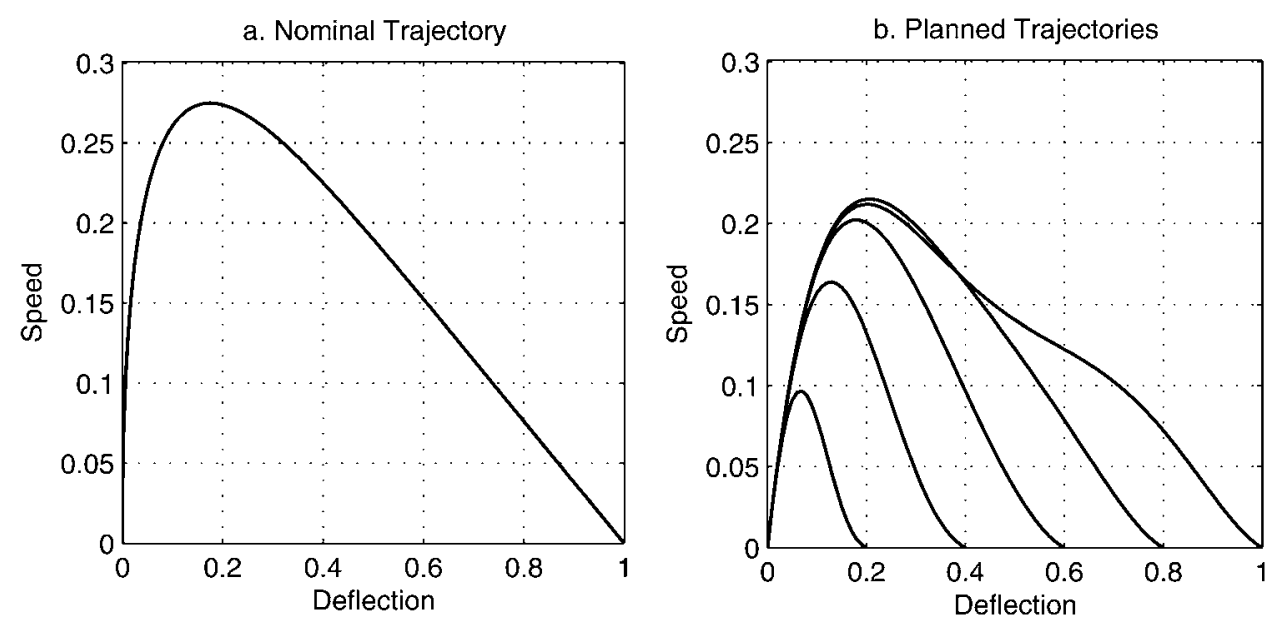

Fig. 5. Nominal and planned trajectories in the normalized coordinates.

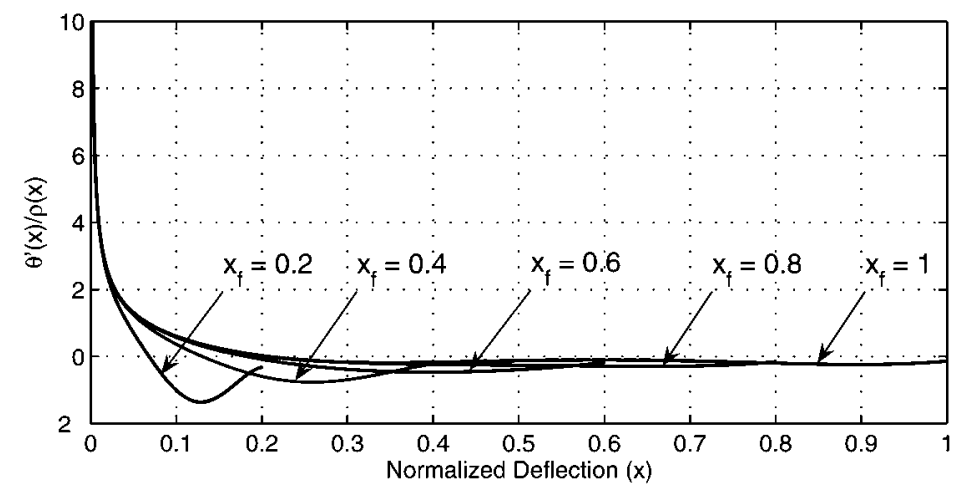

Fig. 6. $\theta^{\prime}(x) / \rho(x)$ for different trajectories.

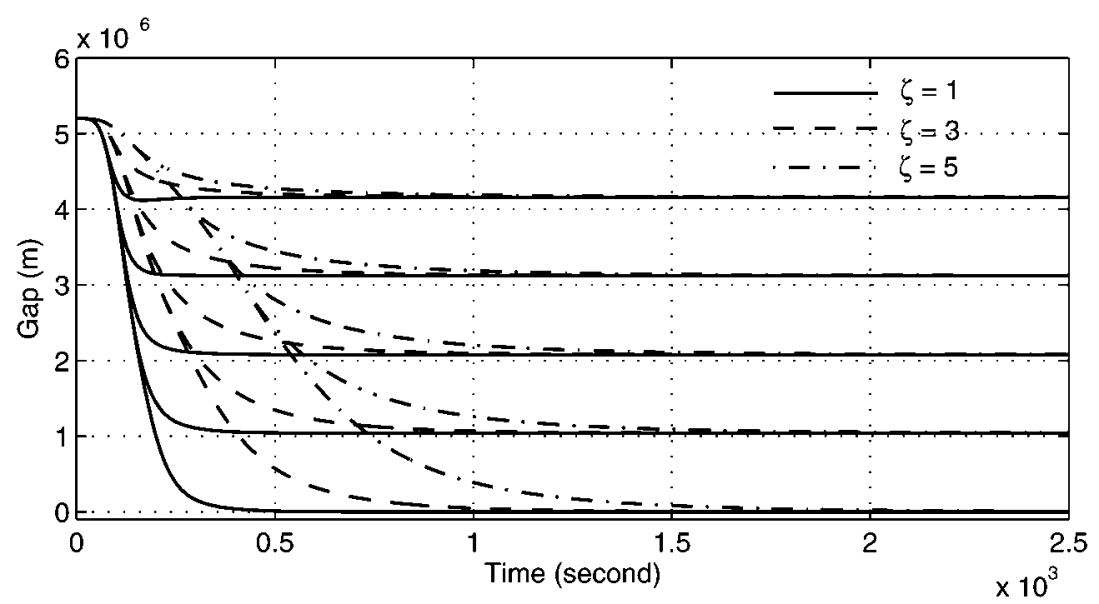

Fig. 7. Responses of voltage controlled parallel-plate electrostatic actuators with system parameter variations.

value) and the damping ratio is fixed to 3 . The estimated resistance is $500 \mathrm{M} \Omega$. A pulse voltage with a width of $0.5 \mathrm{~ms}$ and an amplitude of 2 times of pull-in voltage is applied to steer the operation. The actuator is supposed to be driven by a bipolar voltage source, whose amplitude is limited to \pm 2 times of pull-in voltage by a saturator.

Fig. 7 shows the responses of actuators controlled by the proposed scheme corresponding to the deflections of $20 \%$, $40 \%, 60 \%, 80 \%$, and $100 \%$ of the full gap, respectively. In the simulation, the elastic constant of these devices is set to $388 \mathrm{~N} / \mathrm{m}$ (the measured value) and the damping ratio is $\zeta=1, \zeta=3$, and $\zeta=5$, respectively. It can be seen that the proposed closed-loop control extends the stable operational range to the full gap.

In order to demonstrate the performance of the closed-loop control scheme, systems under the constant voltage control are also simulated and the results are shown in Fig. 8. Since the stable operational range for open-loop control schemes is limited by the pull-in position, only a one third deflection of the full gap is considered. It can be seen that systems with a closed-loop control are much faster than the ones using a constant voltage control scheme. Note that a possible solution to obtain a shorter 


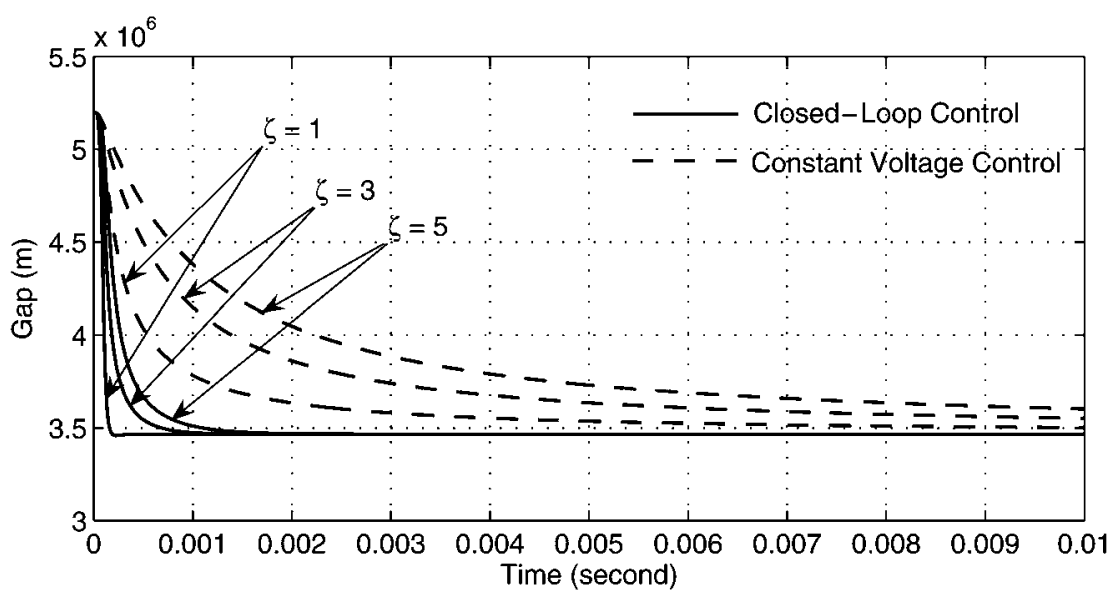

Fig. 8. Closed-loop control versus constant voltage control.

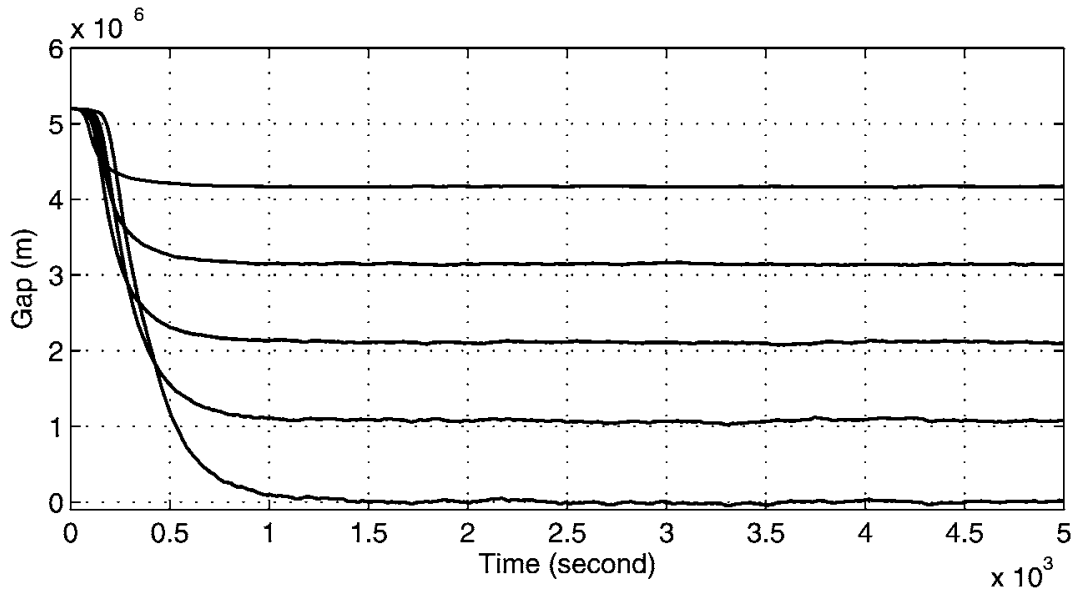

Fig. 9. System responses with noisy measurements.

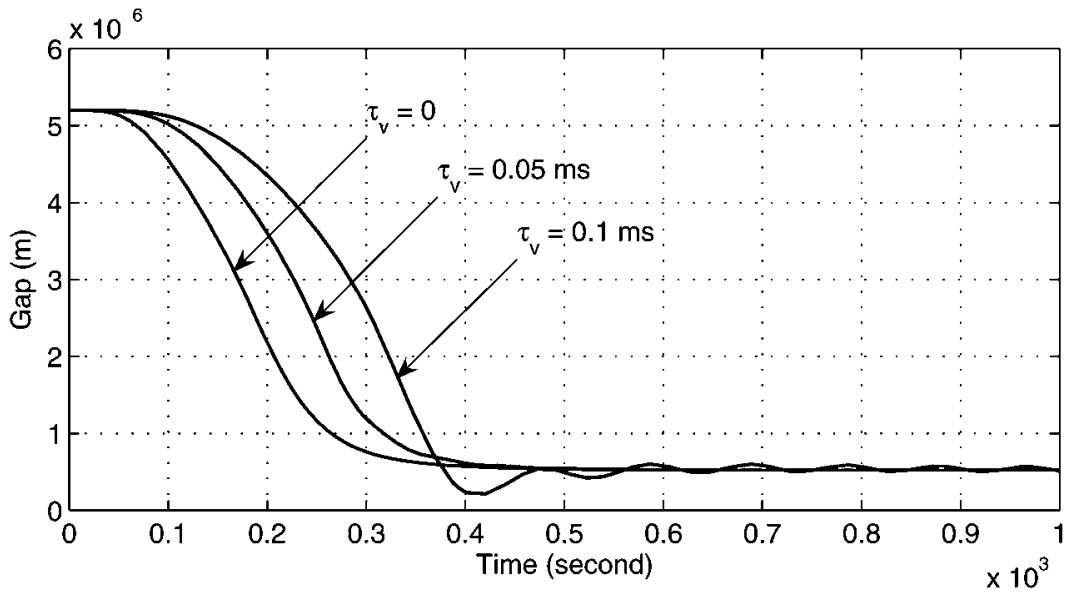

Fig. 10. System responses with control signal delays.

response time is to reduce the viscous damping of the device by putting the mirror into a low pressure chamber. Clearly, closedloop control offers a simpler, more reliable, and less expensive alternative for improving the response time.

The system is also tested against measurement errors, which are modeled as independent zero-mean uniformly distributed noises with maximum amplitude equal to $5 \%$ of the value of setpoint and the corresponding charge, respectively. The influence of measurement noises to the performance is shown in Fig. 9. It has also been observed that the system robustness is decreased due to the presence of measurement noises.

The last test simulated a voltage source delayed by a first order system whose time constant is $\tau_{v}$. Since the slower the system, the bigger the delay can be tolerated, the simulation addressed only the fastest system, namely the one with a damping ratio of 1 . The deflection is set to $90 \%$ of the full gap. Fig. 10 shows the responses corresponding to the control using a voltage source with a time constant $\tau_{v}$ equal to 0.05 and $0.1 \mathrm{~ms}$, or 
equivalently with a response time of 0.2 and $0.4 \mathrm{~ms}$, respectively. For the purpose of comparison, the response of a device driven by an ideal voltage source is also sketched. Note that the response time of the ideal system is about $0.4 \mathrm{~ms}$. It can be seen that delays on control signals degrade the system performance, but the system remains stable when the delay does not exceed the system response time. It has also been observed that further increasing the delay could destabilize the system.

Note that all the reported results are obtained by using the same controller, whose design is based on the nominal plant ( $\zeta=3$ and $k=489 \mathrm{~N} / \mathrm{m}$ ). This shows the robustness of the presented control scheme.

\section{CONCLUSION}

This paper addressed the control of a parallel-plate electrostatic actuator, which is the basic element in MEM-DM for AO applications. It has been shown that this system is differentially flat, and based on this property, a control scheme, combining trajectory planning and nonlinear robust control has been presented. The obtained control system was capable of extending the stable actuation range to the full gap, exhibiting an excellent performance in terms of response time, and was robust vis à vis the dynamical characteristics of the device.

Finally, it is worth noting that, in the original design of the presented DM, the air gap of the actuator was intentionally made more than three times the required deflection for implementing the AO application in order to avoid the pull-in phenomena. In fact, with the proposed control scheme, it is possible to reduce air gap to the one-third of its current dimension. An immediate benefit is that the maximum value of driving voltage can be significantly decreased. Consequently, the device will be more reliable, less expensive, and much easier to operate. This shows the importance of taking into account the control strategy in the early stage of MEMS device design.

\section{ACKNOWLEDGMENT}

The authors gratefully acknowledge the helpful comments and suggestions of Professor L. Saydy of the Department of Electrical Engineering, École Polytechnique de Montréal.

\section{REFERENCES}

[1] N. Doble and D. Williams, "The application of MEMS technology for adaptive optics in vision science," IEEE J. Sel. Topics Quantum Electron., vol. 10, pp. 629-635, May/Jun. 2004.

[2] H. Babcock, "The possibility of compensating astronomical seeing," Publ. Astron. Soc. Pac., vol. 65, pp. 229-236, 1953.

[3] G. Vdovin and S. Middelhoek, "Technology and applications of micromachined silicon adaptive mirrors," Opt. Eng., vol. 36, no. 5, pp. 1382-1390, 1997.

[4] Y.-A. Peter, E. Carr, J. Mansell, and O. Solgaard, "Optical MEMS for adaptive optics applications," in 21st Meet. Electrochem. Soc., First Int. Symp. Integr. Optoelectron., Philadelphia, PA, May 2002, pp. 12-17.

[5] D. C. Dayton, J. D. Mansell, J. D. Gonglewski, and S. R. Restaino, "Novel micromachined membrane mirror characterization and closedloop demonstration," Opt. Commun., vol. 200, no. 1, pp. 99-105, 2001.

[6] P. Kurczynski, H. M. Dyson, B. Sadoulet, J. E. Bower, W. Y.-C. Lai, W. M. Mansfield, and J. A. Taylor, "A membrane mirror with transparent electrode for adaptive optics," in Proc. SPIE MOEMS and Miniaturized Syst. V, 2005, vol. 5719, pp. 155-166.
[7] A. Tuantranont and V. M. Bright, "Segmented silicon-micromachined microelectromechanical deformable mirrors for adaptive optics," IEEE J. Sel. Topics Quantum Electron., vol. 8, no. 1, pp. 33-45, 2002.

[8] N. Doble, M. Helmbrecht, M. Hart, and T. Juneau, "Advanced wavefront correction technology for the next generation of adaptive optics equipped ophthalmic instrumentation," in Proc. SPIE Ophthalmic Technol. XV, 2005, vol. 5688, pp. 125-132.

[9] V. Milanovic, G. A. Matus, and D. T. McCormick, "Gimbal-less monolithic silicon actuators for tiptiltpiston micromirror applications," IEEE J. Sel. Topics Quantum Electron., vol. 10, no. 3, pp. 462-471, 2004.

[10] R. Tyson, Adaptive Optics Engineering Handbook. New York: Marcel Dekker, 1999.

[11] S. Senturia, Microsystem Design. Norwell, MA: Kluwer Academic, 2002.

[12] E. Chan and R. Dutton, "Electrostatic micromechanical actuator with extended range of travel," J. Microelectromech. Syst., vol. 9, pp. $321-328,2000$.

[13] J. Seeger and B. Boser, "Charge control of parallel-plate, electrostatic actuators and the tip-in instability," J. Microelectromech. Syst., vol. 12, pp. 656-671, 2003

[14] P. Chu and K. Pister, "Analysis of closed-loop control of parallel-plate electrostatic microgrippers," in Proc. IEEE Int. Conf. Robot. Autom., 1994, pp. 820-825.

[15] D. Maithripala, J. Berg, and W. Dayawansa, "Capacitive stabilization of an electrostatic actuator: An output feedback viewpoint," in Proc. 2003 Amer. Contr. Conf., Denver, CO, Jun. 4-6, 2003, pp. 4053-4058.

[16] _ "Nonlinear dynamic output feedback stabilization of electostatically actuated MEMS," in Proc. 42nd IEEE Conf. Decision and Control, Maui, HI, Dec. 2003, pp. 61-66.

[17] J. Bryzek and E. Abbott, "Control issues for MEMS," in Proc. 42nd IEEE Conf. Decision and Control, Maui, HI, Dec. 2003, pp. 3039-3047.

[18] J. Lévine, L. Praly, and E. Sedda, "On the control of an electromagnetic actuator of valve positionning on a camless engine," in Proc. AVEC 04 Arnhem, The Netherlands, Aug. 2004.

[19] M. Fliess, J. Lévine, P. Martin, and P. Rouchon, "Flatness and defect of non-linear systems: Introductory theory and examples," Int. J. Contr., vol. 61, pp. 1327-1361, 1995.

[20] M. Fliess and J. Lévine, "A Lie-Bäcklund approach to equivalence and flatness of nonlinear systems," IEEE Trans. Autom. Control, vol. 44, pp. 922-937, 1999

[21] A. Andronov, A. Vitt, and S. Khaikin, Theory of Oscillators. New York: Dover, 1987.

[22] J.-M. Coron, L. Praly, and A. Teel, , Isidori, Ed., "Feedback stabilization of nonlinear systems: Sufficient conditions and Lyapunov and input-output techniques," in Trends in Control. New York: SpringerVerlag, 1995, pp. 293-347.

[23] J. Pont-Nin, A. Rodríguez, and L. Castañer, "Voltage and pull-in time in current drive of electrostatic actuators," J. Microelectromech. Syst., vol. 11, pp. 196-205, 2002.

[24] A. Isidori, Nonlinear Control Systems, 3rd ed ed. London, U.K. Springer-Verlage, 1995.

[25] Y.-A. Peter, E. Carr, and O. Solgaard, "Segmented deformable micromirror for free-space optical communication," in Int. Conf. Optical MEMS and Their Applicat., 2002, pp. 197-198.

[26] D. Koester, R. Mahadevan, B. Hardy, and K. Markus, MUMPS TM Design Handbook. : Revision 7.0, Cronos Integrated Microsystems, A. JDS Uniphase Company, 2001.

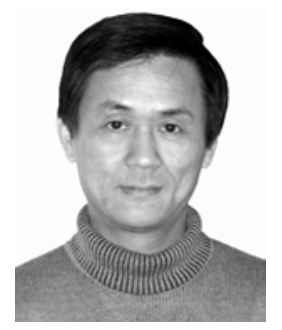

Guchuan Zhu received the M.S. degree in electrical engineering from Beijing Institute of Aeronautics and Astronautics, China, the Ph.D. degree in mathematics and control from the École des Mines de Paris, France, and the graduate diploma in computer science from Concordia University, Montréal, Canada, in 1982, 1992, and 1999, respectively.

During 1982-1989, he was a Lecturer with the Department of Electronic Engineering, Beijing Institute of Aeronautics and Astronautics. He was a Postdoctoral Researcher and a Research Fellow from 1996 to 2000 at the École de Technologie Supérieure, Montréal. During 2000-2004, he worked as a Software Designer at SR Telecom Inc., Montréal. He is now an Assistant Professor with the Department of Electrical Engineering at the École Polytechnique de Montréal. His current research interests include nonlinear system control with applications to microsystems. 


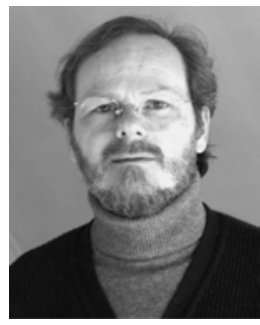

Jean Lévine received the "Doctorat de 3ème cycle" and the "Doctorat d'État" in 1976 and 1984, respectively, from the University Paris-Dauphine, France.

$\mathrm{He}$ is with the Centre Automatique et Systèmes (Systems and Control Center) of the École des Mines de Paris since 1975. His fields of interest include the theory and applications of nonlinear control, and in particular differential flatness. He has worked on many industrial applications such as distillation columns, chemical reactors, food and bioengineering processes, aircraft control, car equipments, cranes, machine tools, magnetic bearings, and high-precision positioning systems.

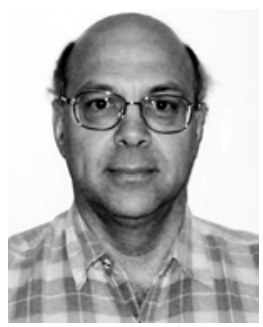

Laurent Praly graduated from École Nationale Superieure des Mines de Paris, France, in 1976.

After working in industry for three years, he joined the Centre Automatique et Systèmes at École des Mines de Paris in 1980. From July 1984 to June 1985, he spent a sabbatical year as a Visiting Assistant Professor with the Department of Electrical and Computer Engineering, University of Illinois at Urbana-Champaign. Since 1985, he has continued with the Centre Automatique et Systèmes where he served as Director for two years. His main interest is in feedback stabilization of controlled dynamical systems under various aspects-linear and nonlinear, dynamic, output, under constraints, with parametric or dynamic uncertainty. On these topics he is contributing both on the theoretical aspect with many academic publications and the practical aspect with applications in power systems, mechanical systems, aerodynamical, and space vehicles.

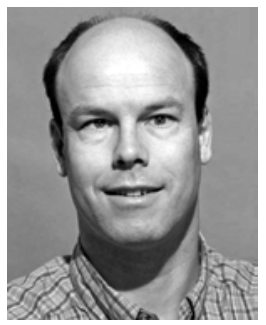

Yves-Alain Peter (M'03) received the Diploma in physics and the Ph.D. degree in 1994 and 2001, respectively, from the University of Neuchâtel, Switzerland.

From 2001 to 2003, he was a Postdoctoral Researcher with Stanford University, Stanford, CA. From 2003 to 2004, he was a R\&D Engineer and Project Leader with the Swiss Center for Electronics and Microtechnology (CSEM), Neuchâtel. He is now an Assistant Professor and Associate Director of the Microfabrication Laboratory, Engineering Physics Department, École Polytechnique de Montréal, Canada. His research interests are micro- and nanooptoelectromechanical systems with applications in adaptive optics and tunable nanophotonics structures.

Dr. Peter is member of the Optical Society of America (OSA) and of the Swiss Physical Society. 